Enfoques. Revista de Investigación en Ciencias de la Administración http://doi.org/10.33996/revistaenfoques.v5i17.104

\title{
ESTUDIO DE TIEMPOS Y SU RELACIÓN CON LA PRODUCTIVIDAD
}

\author{
TIME STUDY AND ITS RELATION TO PRODUCTIVITY \\ ESTUDO DO TEMPO E SUA RELAÇÃO COM A PRODUTIVIDADE
}

Angie Mabel Muñoz Choque

Artículo recibido octubre 2020 | Arbitrado noviembre 2020 | Publicado 01 de enero 2021

\section{Resumen}

Los factores que afectan la productividad suelen variar en cada industria, en este sentido, la investigación el propósito del estudio fue plantear acciones para incrementar la productividad del sector de despacho en una fábrica de cemento boliviana, con base en el estudio de tiempos. Este estudio de alcance relacional tiene base en la interdependencia entre productividad (desempeño de operarios y eficiencia de máquinas) y tiempos de operación, condiciones de trabajo y mantenimiento, las técnicas empleadas fueron: observación participante, revisión bibliográfica, entrevista y cronometraje. Como resultado se plantearon dos acciones para elevar la productividad, relacionadas con reducción de tiempos improductivos y mantenimiento preventivo. Sin embargo, dado que se descartó la existencia de correlación entre productividad y condiciones de trabajo, no se platearon acciones en este sentido. Se concluye que, en contextos particulares, las variables que afectan la productividad varían, el estudio de tiempos permitió plantear acciones específicas para la empresa estudiada.

Palabras clave: Productividad; industria; cemento; tiempo, despacho

\author{
Angie Mabel Muñoz Choque \\ angiemcind@gmail.com \\ Orcid: 0000-0002-0517-6246 \\ Universidad Andina Simón Bolívar, \\ Bolivia. \\ Ingeniero Industrial. Magíster en \\ Administración de Empresas, de la \\ Universidad Andina Simón Bolívar. \\ Diplomado en Big Data Analytics \\ \&amp; Data Science, de la Universidad \\ Privada Boliviana (UPB). Diplomado en \\ Educación Superior y Consultor Junior \\ en Seguridad Industrial, Salud e \\ Higiene Ocupacional y Gestión \\ Ambiental. Universidad Andina Simón \\ Bolívar, Bolivia
}

\begin{abstract}
The factors that affect productivity tend to vary in each industry, in this sense, the research purpose of the study was to propose actions to increase the productivity of the dispatch sector in a Bolivian cement factory, based on the study of times. This relational scope study is based on the interdependence between productivity (operator performance and machine efficiency) and operating times, working conditions and maintenance, the techniques used were: participant observation, bibliographic review, interview and timing. As a result, two actions were proposed to increase productivity, related to reducing unproductive times and preventive maintenance. However, since the existence of a correlation between productivity and working conditions was ruled out, no action was taken in this regard. It is concluded that, in particular contexts, the variables that affect productivity vary, the study of times allowed to propose specific actions for the company studied.
\end{abstract}

Key words: Productivity; industry; cement; time, dispatch 


\section{Resumo}

Os fatores que afetam a produtividade tendem a variar em cada indústria, nesse sentido, o objetivo da pesquisa do estudo foi propor ações para aumentar a produtividade do setor de despacho em uma fábrica de cimento boliviana, com base no estudo de tempos. Este estudo de escopo relacional baseia-se na interdependência entre produtividade (desempenho do operador e eficiência da máquina) e tempos de operação, condições de trabalho e manutenção, as técnicas utilizadas foram: observação participante, revisão bibliográfica, entrevista e tempo. Como resultado, foram propostas duas ações para aumentar a produtividade, relacionadas à redução dos tempos improdutivos e à manutenção preventiva. No entanto, uma vez que foi excluída a existência de correlação entre produtividade e condições de trabalho, nenhuma ação foi tomada a este respeito. Conclui-se que, em contextos particulares, as variáveis que afetam a produtividade variam, o estudo dos tempos permitiu propor ações específicas para a empresa estudada.

\section{INTRODUCCIÓN}

La productividad es fundamental para cualquier empresa y su adecuada gestión es de gran preocupación para los tomadores de decisiones, es por lo que la aplicación de técnicas modernas que optimicen los procesos de producción es una excelente oportunidad para ofrecer resultados tangibles de mejora. Esta variable cobra todavía más importancia en la industria del cemento, ya que se trabaja con recursos no renovables. En Bolivia la industria cementera es un sector cada vez más competitivo, se observa que en los últimos años las empresas cruceñas están ganando liderazgo en el mercado. Esto pone en peligro a empresas del sur que, si bien tienen antigüedad, no han logrado alcanzar altos niveles de competitividad.

Es el caso de la Fábrica Nacional de Cemento Sociedad Anónima (FANCESA), que fue fundada en 1948 en el departamento de Chuquisaca y actualmente oferta 3500 toneladas de cemento al día a nivel nacional (FANCESA, 2015). El proceso productivo en esta empresa inicia desde la extracción de la piedra caliza en el sector de cantera, posteriormente pasa a trituración, molino de crudo, calcinación, molino de cemento $y$ despacho, donde ya se obtiene el producto terminado.

Esta fábrica presenta en algunos sectores cierta limitación en su capacidad productiva, los cuales son considerados como cuellos de botella en el proceso, por lo que cualquier demora en estas áreas repercute inmediatamente en la productividad de la planta, tal es el caso del sector de despacho (FANCESA, 2019). La mejora continua de este sector se ve afectada entre otras cosas, por tiempos improductivos, lo que ocasiona que no se aproveche la capacidad instalada y la mano de obra disponible en su totalidad.

$\mathrm{Si}$ bien se van realizando permanentemente proyectos con el objetivo de incrementar su capacidad productiva, no obstante persisten algunas dificultades que afectan su rendimiento, es por eso que se ve la necesidad de realizar un análisis sistemático para proponer opciones enfocadas a elevar la productividad en el sector de despacho a fin de buscar oportunidades de mejora en el mismo.

Surge entonces la pregunta ¿cómo aportar a la mejora de la productividad del sector de despacho?, en respuesta a la interrogante nació el objetivo del estudio destinado a proponer acciones para elevar la productividad del sector de despacho en la FANCESA, con base en el estudio de tiempo de producción. 
La importancia radica en la magnitud que tiene la Fábrica a nivel departamental, con una participación de mercado aproximada al $28 \%$ en el ámbito cementero nacional y elevada influencia en el incremento del PIB del departamento de Chuquisaca (FANCESA, 2019). Se ve la necesidad social de aportar, desde una perspectiva científica, al incremento de su productividad. La investigación que se presenta es una oportunidad de generar beneficios para la empresa y para todo el departamento.

A continuación, se hace referencia al sustento teórico usado en el estudio, en relación con productividad, estudio de tiempos.

\section{Productividad}

Los resultados obtenidos por una empresas deben ser medidos a partir de indicadores que reflejen aspectos tales como: rentabilidad, eficiencia, eficacia, productividad, entre otros; que permita medir su nivel de competitividad nacional e internacional y retroalimentar la gestión de sus procesos a fin de que la economía crezca y sea capaz de sostener una mejor calidad de vida. (Sira, 2011). La productividad es el nivel de rendimiento con que se emplean los recursos, la relación entre producción e insumo para crear valor agregado (Heizer, 2009). Así, las empresas disponen de recursos con los que crea el producto deseado y que determinan la productividad: terreno, edificios, materiales, energía, máquina, equipo y factor humano. Sin embargo, se debe considerar también las condiciones y medio ambiente de trabajo. (Kanawaty, 2012).

\section{Estudio de tiempos, estudio del trabajo}

El estudio de tiempos nace en el siglo XVIII con el francés Perronet, sesenta años más tarde, Babbage aplicó el estudio de tiempos a análisis de los alfileres, descubriendo que una libra de alfileres debía fabricarse en exactamente 7,6892 horas, así él aportó de esta manera a elevar la productividad de la industria. Por su parte, Taylor a inicios del siglo $\mathrm{XX}$, diseñó métodos de trabajo en los que el hombre y la máquina eran una unidad, esto funcionaba así: el hombre estaba inspirado por el salario y esto producía que de un uso eficiente a la máquina de acuerdo con instrucciones dadas. Los principios principios científicos son: ley de integración, ley de división del trabajo y ley de armonía (Marcombo, 2015).

El estudio de tiempos aplica técnicas para determinar el tiempo en que se lleva a cabo una operación, según una norma o método establecido. Considera la fatiga, las demoras personales y los retrasos inevitables, (Gonzales, 2014).

El estudio de trabajo comprende varias técnicas, pero en especial son de mayor uso el estudio de métodos y la medición de trabajo. El estudio de métodos es el registro y examen crítico sistemático de los modos de realizar actividades, con el fin de efectuar mejoras, el enfoque básico del estudio de métodos consiste en el seguimiento de ocho etapas o pasos: seleccionar, registrar, examinar, establecer, evaluar, definir, implantar y controlar (Kanawaty, 2012).

Según Ruiz (2013), el control de la productividad está totalmente asumido y es sugerido para organizaciones que tienen altos 
estándares de calidad, por medio de esta práctica se tendrá una herramienta de mejora continua, se estima que las desviaciones en los costes y tiempos de producción se darán por grupos de causales: a) las incidencias causadas por problemas en la gestión y b) un desempeño por debajo del normal. En ambos casos el estudio de tiempos es clave para determinar la causa.

Es posible medir la productividad según el grado de eficiencia con que se aplican recursos humanos y materiales, así es importante medir el grado de eficiencia. Para esto, un modelo de programación de la producción proponer reducir el tiempo de terminación del último trabajo aplicando una meta. Otro método, es el estudio de tiempos y movimientos para asignar de forma óptima las tareas a los operarios. Para este último método se deberá contar con un informe detallado de sus actividades, para analizarlas y mejorarlas, (Morillo Santa Cruz, 2007).

\section{Mejora continua}

Es un proceso infinito que comprende: personas, equipo, proveedores, materiales y procedimientos, en busca de erradicar ineficiencias en un sistema de producción. Estadísticamente, en las organizaciones sin gestión de mejora continua el volumen de la ineficiencia puede estar entre un 15 y $25 \%$, ocasionando un efecto negativo en la productividad. (García, 2007). La base de esta filosofía es que cada aspecto de una operación puede ser mejorado, según Heizer, 2009, la meta es la perfección, la cual nunca se alcanza pero siempre se busca.
Por tanto, las organizaciones lograrán el liderazgo en la medida en que se acercan a la excelencia en cada uno de sus procesos, con orientación a la mejora continua. Para esto es útil tener un sistema de gestión que este claramente orientado a los procesos (García, 2009; Andrade, 2018).

\section{METODOLOGÍA}

Se trata de un estudio con enfoque mixto y alcance relacional, tuvo como objetivo relacionar el estudio de tiempos con la productividad, ambas variables en sus respectivas dimensiones fueron tratadas como numéricas y nominales. El estudio del trabajo se desglosó en tres etapas: registro de hechos, estudio de métodos y medición de tiempos. Consecuentemente, se abordó el estudio de la productividad midiendo el desempeño de cada operario y la eficiencia de cada máquina.

Con estos fines, se usó la técnica de la observación indirecta, participante, estructurada y de campo, para controlar y cronometrar los tiempos de operación tanto de la maquinaria como de los operadores y su desempeño. También ayudó con el registro de los movimientos que realizan los operarios en sus diferentes actividades. De la misma manera, se aplicaron entrevistas temáticas personales al jefe de producción, encargado mecánico, encargado eléctrico, jefe de recursos humanos y operadores del área. También se hizo una revisión bibliográfica de datos estadísticos del periodo 2017-2019. El siguiente cuadro muestra la herramienta específica usada para cada propósito. 
Tabla 1. Instrumentos aplicados según pasos y dimensiones

\begin{tabular}{|c|c|c|c|}
\hline $\begin{array}{l}\text { DIMENSIONES } \\
\text { /ETAPAS }\end{array}$ & INDICADORES & $\begin{array}{c}\text { TIPO DE } \\
\text { VARIABLE }\end{array}$ & $\begin{array}{l}\text { INSTRUMENTO } \\
\text { /TÉCNICA }\end{array}$ \\
\hline $\begin{array}{l}\text { Desempeño del } \\
\text { Operario }\end{array}$ & $\begin{array}{l}\text { Trabajo realizado por operario } \\
\text { regular/unidad de tiempo } \\
\text { Trabajo realizado por operario } \\
\text { capacitado/unidad de tiempo }\end{array}$ & Continua & $\begin{array}{l}\text { Cronometraje } \\
\text { Guía de observación }\end{array}$ \\
\hline $\begin{array}{l}\text { Eficiencia } \\
\text { la máquina }\end{array}$ & $\begin{array}{l}\text { Capacidad real vs. capacidad } \\
\text { nominal }\end{array}$ & Continua & $\begin{array}{l}\text { Cronometraje } \\
\text { Guía de Observación }\end{array}$ \\
\hline \multirow{5}{*}{ Registrar hechos } & $\begin{array}{l}\text { Datos históricos de la } \\
\text { planificación de producción }\end{array}$ & Continua & Datos 2017,2018 y 2019 \\
\hline & $\begin{array}{l}\text { Datos Históricos de la } \\
\text { planificación de despacho de } \\
\text { bolsas de cemento, big bag y } \\
\text { cisterna }\end{array}$ & Continua & Datos 2017,2018 y 2019 \\
\hline & $\begin{array}{l}\text { Capacidad global del despacho } \\
\text { diario }\end{array}$ & Continua & $\begin{array}{l}\text { Observación } \\
\text { Datos diarios } 2019\end{array}$ \\
\hline & $\begin{array}{l}\text { Actividades que presentan } \\
\text { tiempos improductivos }\end{array}$ & Nominal & $\begin{array}{l}\text { Entrevista al Jefe de } \\
\text { Producción, Mecánicos, } \\
\text { Eléctricos, Operarios }\end{array}$ \\
\hline & Frecuencia de horas extras & Ordinal & $\begin{array}{l}\text { Entrevista al Jefe de } \\
\text { Producción, Operarios y } \\
\text { RRHH }\end{array}$ \\
\hline Estudio de métodos & $\begin{array}{l}\text { Movimientos que realiza el } \\
\text { operario en embolsado, big bag, } \\
\text { cisterna }\end{array}$ & Ordinal & Observación \\
\hline $\begin{array}{l}\text { Medición } \\
\text { tiempos }\end{array}$ & Bolsas de cemento/ minutos & Continua & $\begin{array}{l}\text { Cronometraje } \\
\text { Guía de observación }\end{array}$ \\
\hline
\end{tabular}

El estudio del método se realizó siguiendo 6 pasos. Primero se seleccionó el trabajo para el estudio, con miras a mejorar la manera en que se realiza, tomando en cuenta consideraciones económicas o de eficiencia en función de costos, consideraciones técnicas y recursos humanos. Como segundo paso se registraron todas las actividades, tareas $\mathrm{y}$ hechos relacionados al trabajo, como base para hacer el examen crítico y para idear el método perfeccionado. El tercer paso consistió en examinar los métodos utilizados, el modo en que se realizó el trabajo, su propósito, el lugar, la secuencia en que se llegó a cabo. El cuarto paso se enfocó a definir el método más práctico, económico y eficaz, recibiendo aporte del personal experto. A continuación, quinto paso, se evaluó las opciones de mejora o cambio de método y se hizo una medición de la relación costo/eficacia entre ambos métodos. Finalmente, se presentó el nuevo método. 
Para el estudio del método se aplicó técnica de la observación participativa, es decir, el investigador trabajó junto con los operadores de las máquinas y todo el equipo fue capacitado y tuvo conocimiento sobre los objetivos. De esta manera fue posible seleccionar al operario, analizar el trabajo y desglosarlo en sus elementos, registrar los valores elementales de los tiempos transcurridos, calificar el desempeño del operario y asignar los suplementos $u$ holguras adecuadas.

Posteriormente, se ajustaron los tiempos normales de las tareas mediante el sistema de calificación del desempeño que permitió evaluar la efectividad con base a un operario calificado. Se evaluaron cuidadosamente factores como la velocidad, destreza, movimientos falsos, ritmo, coordinación y otros según el tipo de tarea.

La fórmula usada para ajustar el tiempo medio observado (TO) fue:

- $\mathrm{TN}=\mathrm{TO} \times \mathrm{C} / 100$

- Dónde:

- TO es el tiempo medio observado

- TN es el tiempo normal

C es la calificación del desempeño del operario expresada como porcentaje

El tiempo estándar (TE), se calculó de la siguiente manera:

$$
\mathrm{TE}=\mathrm{TN} /(1-\text { holgura })
$$

Los tiempos calculados consideraron la adición de suplementos $u$ holguras que se pudieran presentar en la sección, es decir viajes al baño, comidas, interrupciones por el supervisor, variación de material, etc. Para la realización del estudio de tiempos, se utilizó una escala de medición en minutos y se utilizó un cronómetro manual.

Finalmente, se platearon 3 hipótesis, específicas para su comprobación, se trabajó con pruebas no paramétricas, y el coeficiente de correlación de Spearman por presentar tanto variables de razón como variables nominales y no presentar una distribución normal.

\section{RESULTADOS}

Los siguientes párrafos están destinados a mostrar los principales resultados obtenidos por medio de la aplicación de 3 técnicas: a) observación indirecta, participante, estructurada y de campo, b) entrevistas temáticas personales al jefe de producción, encargado mecánico, encargado eléctrico, jefe de recursos humanos y operadores del área y c) revisión bibliográfica de datos estadísticos del periodo 2017-2019.

En el estudio se pudo evidenciar que no existía una planificación sistemática para el mantenimiento del sector, por el contrario, lo que se realiza generalmente es un mantenimiento correctivo, es decir, que se atiende los desperfectos que necesitan ser revisados para continuar la rutina de trabajo, provocando una paralización del proceso y generando pérdidas por el tiempo invertido y los gastos generados. Además, si bien la empresa cuenta con personal capacitado para reparar las fallas mecánicas en el mínimo tiempo posible, este departamento es un grupo bastante sectorizado, entendiendo que, si los encargados mecánicos del sector de despacho están ocupados realizando otras tareas y no se encuentran disponibles, los operarios mecánicos de otra área no son destinados a solucionar el problema de esta área, provocando más tiempos improductivos.

La empresa no cuenta con una estandarización de procesos, los empleados saben lo que deben hacer, pero lo realizan de la forma que ellos consideran se más 
adecuada, por lo que se presenta bastante variabilidad en los procesos, ocasionando la presencia de tiempos improductivos.

La Fábrica estudiada es consciente de la importancia de estandarizar y mejorar sus procesos productivos sistemáticamente, con el fin de responder a las exigencias del mercado, incidir positivamente en la rentabilidad financiera de la empresa, minimizar los tiempos de operación y seguir consolidándose como una de las mejores empresas cementeras del país, ya que un proceso organizado se traduce en mejora continua.

El proceso de despacho consta de tres modalidades: Ensacado que representa 95\% de la producción, big bag que cubre $4 \%$ y Cisterna que solamente representa $1 \%$.

La modalidad de ensacado cuenta con 15 empleados para cada turno, operando actualmente tres turnos de trabajo para minimizar la cantidad de horas extras. Sin embargo, el área presenta permanentemente mayor cantidad de horas extras respecto a los demás sectores. El área evidencia un desempeño total de sus trabajadores del $65 \%$ y una eficiencia de las ensacadoras del $85 \%$, no empleando así su capacidad total. Por otra parte, en la evaluación de la producción de bolsas de cemento por mes de las ensacadoras de 8 y 6 pitones con la capacidad de producción de ambas se obtiene una diferencia considerable de entre el $24 \%$ y $39 \%$ de pérdidas en horas de operación debido principalmente a paradas imprevistas. por lo que se debe prestar especial atención a esto para alcanzar una mejora en la productividad de dicha área. Su proceso productivo comprende los siguientes pasos: preselección de bolsas, traslado de bolsas, acomodo de bolsas, levantamiento de bolsas, colocado de bolsas, llenado de bolsas en cada pitón, transporte de bolsas blanda, sellado de bolsas, transporte de bolsa, descargo de bolsa y control de peso.

En cuanto a la modalidad big bag, esta cuenta con diez empleados y un solo turno de trabajo, el desempeño de estos es del $75 \%$ aproximadamente. De la evaluación realizada a la producción de bolsas por mes con su capacidad de producción, tomando en cuenta sus horas de operación, se obtienen una diferencia de entre el $7 \%$ y $25 \%$ de pérdidas en horas de operación, sin embargo existieron meses donde la producción de bolsas de big bag alcanzó la capacidad de la máquina. lo que indica que esta modalidad no precisa de la misma atención que la de ensacado.

La modalidad de despacho mediante cisterna no cuenta con personal fijo, tampoco cuenta con un turno de trabajo específico, por lo que su participación en este análisis no es significativa.

En cuanto al análisis de la actividad productiva para las tres modalidades, se tomaron en cuenta las siguientes variables en el estudio: a) desempeño de los trabajadores, b) capacidad nominal, c) capacidad nominal a velocidades programadas, d) capacidad real, e) porcentaje de utilización de equipo, f) capacidad nominal bolsas/min, g) capacidad máxima evidenciada, h) capacidad mínima evidenciada, i) promedio de eficiencia de pitones $y, j$ ) pérdidas en horas de operación por paradas imprevistas. En la tabla 2 se presenta una síntesis información recogida en relación a estas variables. 
Tabla 2. Síntesis de la actividad productiva para las modalidades: ensacado, big bag y cisterna.

\begin{tabular}{|c|c|c|c|c|}
\hline \multirow[b]{2}{*}{ Detalle } & \multicolumn{2}{|c|}{ Ensacado Sacos de 50 kg. } & \multirow[b]{2}{*}{ Big bag } & \multirow[b]{2}{*}{ Cisterna } \\
\hline & $\begin{array}{c}\text { Ensacadora de } 6 \\
\text { pitones }\end{array}$ & $\begin{array}{c}\text { Ensacadora de } 8 \\
\text { pitones }\end{array}$ & & \\
\hline $\begin{array}{l}\text { Desempeño de los } \\
\text { trabajadores }\end{array}$ & $65 \%$ & & $75 \%$ & \\
\hline Capacidad nominal & 2.000 bolsas/hora & $\begin{array}{l}2400 \\
\text { bolsas/hora }\end{array}$ & - & \\
\hline $\begin{array}{l}\text { Capacidad nominal a } \\
\text { velocidades } \\
\text { programadas }\end{array}$ & 1.560 bolsas/hora & $\begin{array}{l}2160 \\
\text { bolsas/hora }\end{array}$ & - & \\
\hline Capacidad real & 1.380 bolsas/hora & $\begin{array}{l}1860 \\
\text { bolsas/hora }\end{array}$ & - & \\
\hline $\begin{array}{l}\text { Porcentaje de } \\
\text { utilización de equipo }\end{array}$ & $88.50 \%$ & $86.10 \%$ & - & $A$ \\
\hline $\begin{array}{l}\text { Capacidad nominal } \\
\text { bolsas/min }\end{array}$ & 26 bolsas/min & 35 bolsas/min & - & requerimiento \\
\hline $\begin{array}{l}\text { Capacidad máxima } \\
\text { evidenciada }\end{array}$ & 24 bolsas/min & 33 bolsas/min & 1 bolsa / $2.9 \mathrm{~min}$ & \\
\hline $\begin{array}{l}\text { Capacidad mínima } \\
\text { evidenciada }\end{array}$ & 12 bolsas/min & 22 bolsas/min & 1 bolsa / $3.01 \mathrm{~min}$ & \\
\hline $\begin{array}{l}\text { Promedio de eficiencia } \\
\text { de pitones }\end{array}$ & $93.48 \%$ & $94.82 \%$ & - & \\
\hline $\begin{array}{l}\text { Pérdidas en horas de } \\
\text { operación por paradas } \\
\text { imprevistas }\end{array}$ & Entre $24 \%-39 \%$ & & Entre $7 \%-25 \%$ & \\
\hline
\end{tabular}

Como se puede observar en la tabla 2, la modalidad cisterna funciona a requerimiento, por tanto, no fue factible evaluar las variables, además como representa una producción mínima no se consideró relevante proceder con un estudio a profundidad. En cuanto a la modalidad ensacado, se evidenció que el comportamiento es similar para 6 u 8 pitones, resalta la posibilidad de elevar la eficiencia en $6 \%$ para ambas maquinarias, asimismo es posible incrementar la utilización de las máquinas hasta en un $14 \%$. Finalmente, en la modalidad big bag, no es posible medir las variables: capacidad nominal, capacidad nominal a velocidades programadas, capacidad real, porcentaje de utilización de equipo, capacidad nominal bolsas/min y promedio de eficiencia de pitones.

Posteriormente, según se muestra en la tabla 3 , se procedió a la verificación estadística de las hipótesis planteadas.

$\mathbf{H O}_{1}$ : La reducción de tiempos de operación, no tendrá efecto positivo en la productividad del área de despacho de FANCESA.

$\mathrm{HO}_{2}$ : Mejorar las condiciones de trabajo en el área de despacho de FANCESA, no mejorará la productividad del área.

$\mathrm{HO}_{3}$ : La existencia de un programa de mantenimiento preventivo, no tendrá efecto positivo en la productividad del área. 
Tabla 3. Comprobación de hipótesis

\begin{tabular}{|c|c|c|c|c|}
\hline $\begin{array}{l}\text { Hipótesis } \\
\text { Nula }\end{array}$ & Variables & Sig. (p) & $\begin{array}{c}\text { Spearman } \\
\text { (Rho) }\end{array}$ & Análisis \\
\hline $\mathrm{HO}_{1}$ & $\begin{array}{l}\text { Productividad- } \\
\text { Reducción de } \\
\text { Tiempos de } \\
\text { Operación }\end{array}$ & ,004 & 0,674 & $\begin{array}{l}\text { Correlación positiva significativa a un } \\
\text { nivel de } 0.01 \text {, se puede afirmar que } \\
\text { existe una asociación conjunta de } \\
\text { ambas variables, en la medida en } \\
\text { que la reducción de tiempos de } \\
\text { operación, mejorarán } \\
\text { productividad. }\end{array}$ \\
\hline $\mathrm{HO}_{2}$ & $\begin{array}{l}\text { Productividad- } \\
\text { Programa de Mant. } \\
\text { Preventivo }\end{array}$ & ,049 & $-0,256$ & $\begin{array}{l}\text { Correlación negativa moderada a un } \\
\text { nivel de } 0.05 \text {, es decir que a medida } \\
\text { que se aplique un programa de } \\
\text { mantenimiento preventivo se } \\
\text { reducirá las horas muertas por } \\
\text { paradas inesperadas, contribuyendo } \\
\text { positivamente a la mejora de la } \\
\text { productividad delárea. }\end{array}$ \\
\hline $\mathrm{HO}_{3}$ & $\begin{array}{l}\text { Productividad- } \\
\text { Condiciones de } \\
\text { Trabajo }\end{array}$ & , 078 & ,229 & No existe evidencia significativa. \\
\hline
\end{tabular}

Los resultados obtenidos para las tres hipótesis según la tabla 3 son explicados a detalle a continuación.

Para $\mathrm{HO}_{1}$, el nivel de significancia es menor a $5 \%$, por tanto, se rechaza la hipótesis nula, es decir, se afirma que la reducción de tiempos de operación tendrá un efecto positivo en la productividad del área de despacho de FANCESA, siendo su relación estadísticamente significativa.

$$
\begin{aligned}
& \text { Sig }<\alpha \\
& 0,004<0,05
\end{aligned}
$$

Por otra parte, el valor de Rho de Spearman es 0,674 , lo que significa que presenta una relación alta y directa.

Para $\mathrm{HO}_{2}$, el nivel de significancia es mayor a $5 \%$, por tanto, se acepta la hipótesis nula, es decir, mejorar las condiciones de trabajo en el área de despacho de FANCESA no mejorará la productividad del área significativamente.

$$
\begin{aligned}
& \text { a Sig }>\alpha \\
& 0,078>0,05
\end{aligned}
$$

Para $\mathrm{HO}_{3}$, el nivel de significancia es menor a $5 \%$, por tanto, se rechaza la hipótesis nula, es decir, se afirma que la implementación de un programa de mantenimiento preventivo si mejorará la productividad del área.

$$
\begin{aligned}
& \text { Sig }<\alpha \\
& 0,049<0,05
\end{aligned}
$$

Por otra parte, el valor de Rho de Spearman da - 0,256, lo que significa que existe una correlación negativa moderada. 
Por lo expuesto, se confirma que es posible lograr mejoras en la productividad del área de despacho de FANCESA enfocando esfuerzos a reducir tiempos de operación y el mantenimiento de las máquinas del sector, esto debido al tiempo muerto que existe por paradas imprevistas, ocasionando una considerable diferencia entre la producción real y la capacidad de producción de las máquinas.

Esto aplica especialmente en las ensacadoras de 8 y 6 pitones, ya que, en las otras modalidades de despacho, es decir big bag y cisterna, la diferencia entre estos conceptos no es significativa.

Se propone entonces mejorar la productividad del sector de despacho y envasado mediante dos acciones a desarrollarse en el área: a) implementación de un programa de mantenimiento mecánico preventivo en el sector $y, b$ ) estandarización de los procesos del área de despacho y envasado.

\section{Acción 1: Implementación de un programa de mantenimiento mecánico preventivo en el sector de despacho y envasado}

Las entradas del sistema de mantenimiento comprenden: los recursos de mantenimiento, requerimientos de producción, activos instalados y mediciones realizadas al sistema. Por su parte, la salida del sistema es el activo o equipo disponible, confiable y bien configurado para lograr la operación planeada del sector de despacho y envasado.

Las actividades del programa de mantenimiento preventivo propuesto estarán conformadas por: mantenimiento autónomo e inspecciones periódicas.

El mantenimiento autónomo implica un conjunto de actividades que se realizan diariamente por todos los trabajadores en los equipos que operan, incluyendo inspección, lubricación, limpieza, cambio de herramientas y piezas, analizando y solucionando problemas del equipo y acciones que conduzcan a mantener el equipo en las mejores condiciones de funcionamiento. Por su parte, las inspecciones periódicas son revisiones a intervalos fijos, independientemente de su estado original, para obtener información útil acerca del estado de las partes del equipo, y así predecir fallas y planear acciones de mantenimiento.

Así mismo, se prevé soporte del programa de mantenimiento que deberá estar conformado por los siguientes formatos: a) inventario de máquinas y equipos (nombre, código, modelo, serie, año de fabricación, documentación técnica, fichas de mantenimiento), b) ficha técnica, c) control de lubricación, d) historia de máquinas y equipos, e) costos de mantenimiento por equipo $y, f$ ) orden de trabajo de mantenimiento.

El gráfico 1 muestra cómo se planteó el procedimiento general del programa de mantenimiento preventivo. 


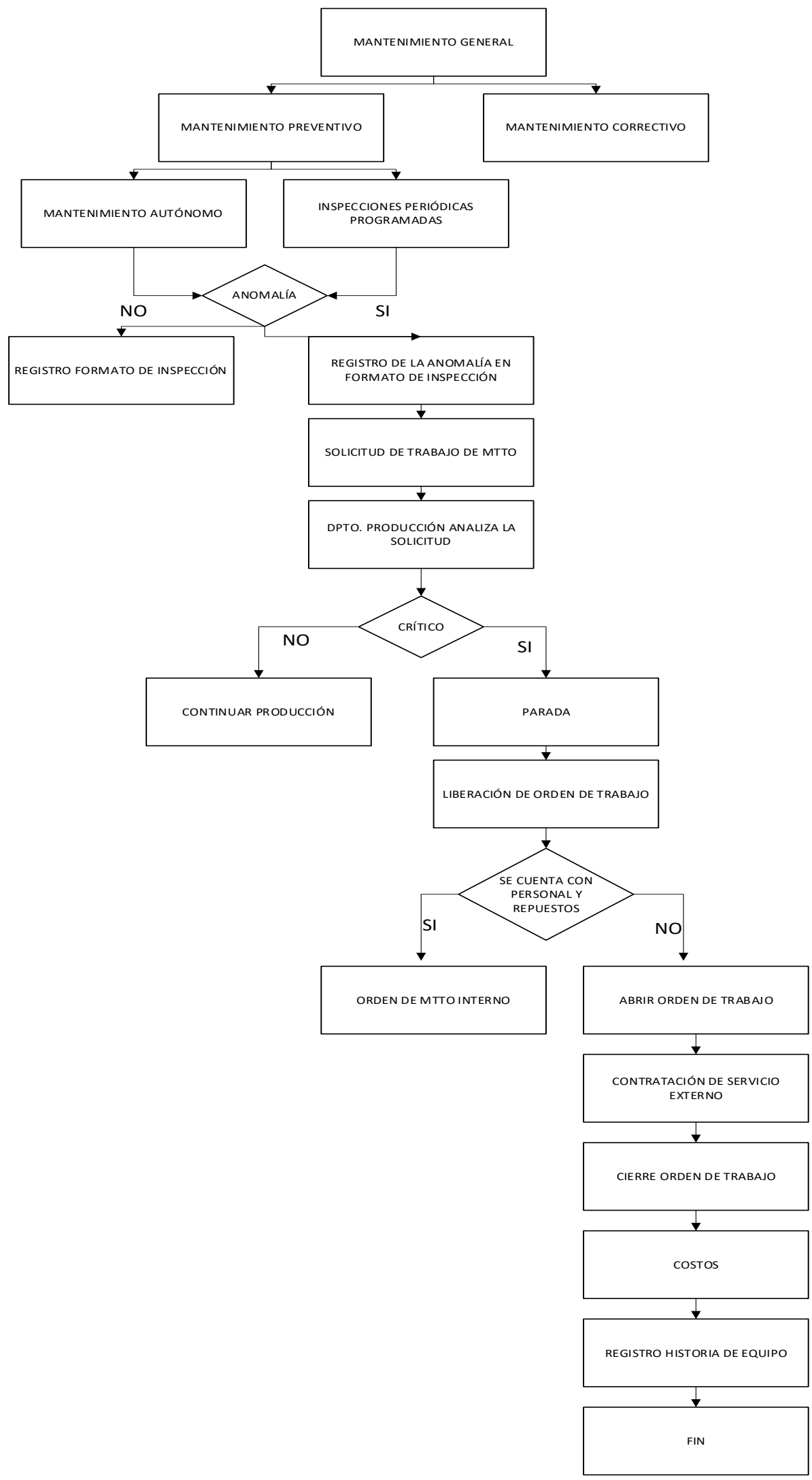

Gráfico 1. Procedimiento general del programa de mantenimiento preventivo 
Como se puede observar en el gráfico 1 , tanto el mantenimiento autónomo como las inspecciones periódicas deben informar anomalías, en caso de no existencia de anomalías se deberá registrar el informe detallando el estado. Por el contrario, en caso de detectar anomalías, los pasos a seguir son 1) registrar 2) solicitar trabajo y 3) la respectiva evaluación de la solicitud por parte del departamento de producción. En caso de ser procedente, se para la producción y se libera la orden de trabajo. Posteriormente, y solo en caso de no contar con personal interno, se abre la orden de trabajo.

Antes de poner en práctica el programa es necesario capacitar y educar a cada una de las personas involucradas, para que en el momento de su ejecución éste alcance el éxito esperado. Asimismo, es importante eliminar en el departamento mecánico la sectorialización que existe actualmente, para que así todo el personal del área pueda atender cualquier problema en las diferentes secciones de la empresa y no así sólo en la que están destinados. Esto además influiría positivamente en la reducción de horas extras del personal encargado del mantenimiento mecánico del sector de despacho y envasado.

\section{Acción 2: Estandarización de procesos} en el área de despacho y envasado de FANCESA.

Al inicio de cada turno se propone destinar 12 minutos para el alistamiento de maquinaria y operarios, y no así $30 \mathrm{~min}$ como es habitual, al finalizar el turno se dedican 6 minutos para hacer entrega de datos del turno al encargado de grupo $y$ hacer empalme con el turno que comienza, por lo tanto en total se emplean en esta actividad 18 minutos.

Con el estudio de tiempos se obtiene las horas ideales de trabajo, al compararlas con las horas reales presentan una diferencia del $19,51 \%$, lo que significaría que es posible una reducción de tiempos de operación en dicho porcentaje. En este sentido, se propuso adecuaciones a los procesos de las ensacadoras de 6 y 8 pitones, en el gráfico 2 se muestra el mapa de procesos para la ensacadora 6 . 


\begin{tabular}{|c|c|c|c|c|c|c|c|c|}
\hline \multicolumn{2}{|c|}{ Mapa de Procesos } & \multicolumn{7}{|c|}{ Operario/Material/Equipo } \\
\hline Ohieto. & Estandarización de & \multicolumn{7}{|c|}{ Actividad } \\
\hline Actividad & & Operación & 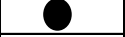 & & & & & \\
\hline Lugar: & Despacho y Envase & Transporte & & & & & & \\
\hline Operarios: & & Espera & 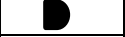 & & & & & \\
\hline \multirow{2}{*}{\multicolumn{2}{|c|}{ ENSACADORA DE 6 PITONES }} & Inspección & $\mathbf{\square}$ & & & & & \\
\hline & & Almacenamiento & $\nabla$ & & & & & \\
\hline \multirow{2}{*}{\multicolumn{2}{|c|}{ Descripción }} & \multirow[t]{2}{*}{ Cantidad } & \multicolumn{2}{|c|}{$\begin{array}{l}\text { Tiempo } \\
\text { (min) }\end{array}$} & \multicolumn{3}{|c|}{ Símbolos } & \multirow[t]{2}{*}{ Observaciones } \\
\hline & & & Ahora & Sistematizado & & $\Rightarrow$ & Q & \\
\hline \multicolumn{2}{|c|}{ Preselección de Bolsas } & 500 Bolsas & 4 & 3 & & & $=$ & \multirow{10}{*}{$\begin{array}{l}\text { Con la estandarización de los } \\
\text { tiempos de operación, el } \\
\text { desempeño de los trabajadores } \\
\text { incrementaría de un } 19,51 \% \\
\text { tomando como base para los } \\
\text { tiempos estandarizados el } \\
\text { tiempo en que realizaría estas } \\
\text { tareas un trabajador calificado. }\end{array}$} \\
\hline & islado de Bolsas & 500 Bolsas & 5 & 4 & & & & \\
\hline & modo de Bolsas & 500 Bolsas & 5 & 3 & & & & \\
\hline Levan & taniento de bolsas & 1 Bolsa & 0,17 & 0,15 & & & & \\
\hline Colocado de & bolsas en boquilla pitón & 1 Bolsa & 0,55 & 0,48 & & & & \\
\hline Llenado c & e bolsas en cada pitón & 33 Bolsas vs $35 \mathrm{BC}$ & 1 & 1 & & & & \\
\hline Sellado c & $\begin{array}{l}\text { le bolsas con fecha de } \\
\text { envasado }\end{array}$ & 1 Bolsa & Automático & Automático & & & & \\
\hline \multicolumn{2}{|c|}{$\begin{array}{c}\text { Transporte de bolsas en banda } \\
\text { transportadora }\end{array}$} & 2 Bolsa & Automático & Automático & & 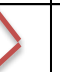 & & \\
\hline \multicolumn{2}{|c|}{ Descargo de Bolsas a Camión } & 1Bolsa & 0,5 & 0,5 & & & & \\
\hline \multirow{2}{*}{\multicolumn{2}{|c|}{ Control de peso de bolsas }} & 1 camión & 15 & 13 & & & + & \\
\hline & & TOTALES & 31,22 & 25,13 & & & & \\
\hline
\end{tabular}

Gráfico 2. Mapa de procesos de la ensacadora de 6 pitones.

En el gráfico 2 se observa la propuesta de tiempos para la estandarización del trabajo realizado en la ensacadora de 6 pitones, se puede evidenciar la notoria diferencia en tiempos de operación entre lo que se realiza actualmente y después de implementar la propuesta.

\begin{tabular}{|c|c|c|c|c|c|c|c|}
\hline \multicolumn{2}{|c|}{ Mapa de Procesos } & \multicolumn{6}{|c|}{ Operario/Material/Equipo } \\
\hline Objeto: & $\begin{array}{c}\text { Estandarización de } \\
\text { procesos }\end{array}$ & \multicolumn{6}{|c|}{ Actividad } \\
\hline Actividad & & Operación & & & & & Dos operarios \\
\hline Lugar: & Despacho y Envase & Transporte & & & & & \\
\hline Operarios: & & Espera & & & & & \\
\hline \multirow{2}{*}{\multicolumn{2}{|c|}{ ENSACADORA DE 8 PITONES }} & Inspección & $\mathbf{\square}$ & & & & \\
\hline & & Almacenamiento & 7 & & & & \\
\hline \multirow{2}{*}{\multicolumn{2}{|c|}{ Descripción }} & \multirow{2}{*}{ Cantidad } & \multicolumn{2}{|c|}{ Tiempo } & \multicolumn{2}{|c|}{ Símbolos } & \multirow{2}{*}{ Observaciones } \\
\hline & & & Ahora & Sistematizado & $\Rightarrow$ & $\mathbf{D}$ & \\
\hline \multicolumn{2}{|c|}{ Preselección de Bolsas } & 500 Bolsas & 6 & 4,5 & & $=$ & \multirow{10}{*}{$\begin{array}{l}\text { Con la estandarización de los } \\
\text { tiempos de operación, el } \\
\text { desempeño de los trabajadores } \\
\text { incrementaría de un } 16,28 \% \text {, } \\
\text { tomando como base para los } \\
\text { tiempos estandarizados el } \\
\text { tiempo en que realizaría estas } \\
\text { tareas un trabajador calificado. }\end{array}$} \\
\hline \multicolumn{2}{|c|}{ Traslado de Bolsas } & 500 Bolsas & 5 & 4 & 7 & & \\
\hline \multicolumn{2}{|c|}{ Acomodo de Bolsas } & 500 Bolsas & 6 & 5 & & & \\
\hline \multicolumn{2}{|c|}{ Levantaniento de bolsas } & 1 Bolsa & 0,17 & 0,15 & & & \\
\hline \multicolumn{2}{|c|}{ Colocado de bolsas en boquilla pitón } & 1 Bolsa & 0,55 & 0,5 & & & \\
\hline \multicolumn{2}{|c|}{ Llenado de bolsas en cada pitón } & 33 Bolsas vs $35 \mathrm{BC}$ & 1 & 1 & & & \\
\hline \multicolumn{2}{|c|}{ Sellado de bolsas con fecha de } & 1 Bolsa & Automático & Automático & & & \\
\hline \multicolumn{2}{|c|}{ Transporte de bolsas en banda } & 2 Bolsa & Automático & Automático & & & \\
\hline \multicolumn{2}{|c|}{ Descargo de Bolsas a Camión } & 1Bolsa & 0,5 & 0,5 & 4 & & \\
\hline \multicolumn{2}{|c|}{ Control de peso de bolsas } & 1 camión & 15 & 13 & & \begin{tabular}{l|l}
+1 \\
\end{tabular} & \\
\hline & & TOTALES & 34,22 & 28,65 & & & \\
\hline
\end{tabular}

Gráfico 3. Mapa de procesos de la ensacadora de 8 pitones. 
Como se puede observar en el gráfico 3, la ensacadora de 8 pitones presenta una diferencia del $16,28 \%$ entre el tiempo que lleva hacer sus tareas a los trabajadores en el proceso regular y el tiempo que toma una vez implementada la propuesta, reduciendo los tiempos improductivos presentes en el área.
La modalidad de despacho mediante big bag presenta menos variabilidad en la ejecución de sus procesos, al ser un único turno la estandarización propuesta fue más sencilla de desarrollar, según se muestra en el gráfico 4.

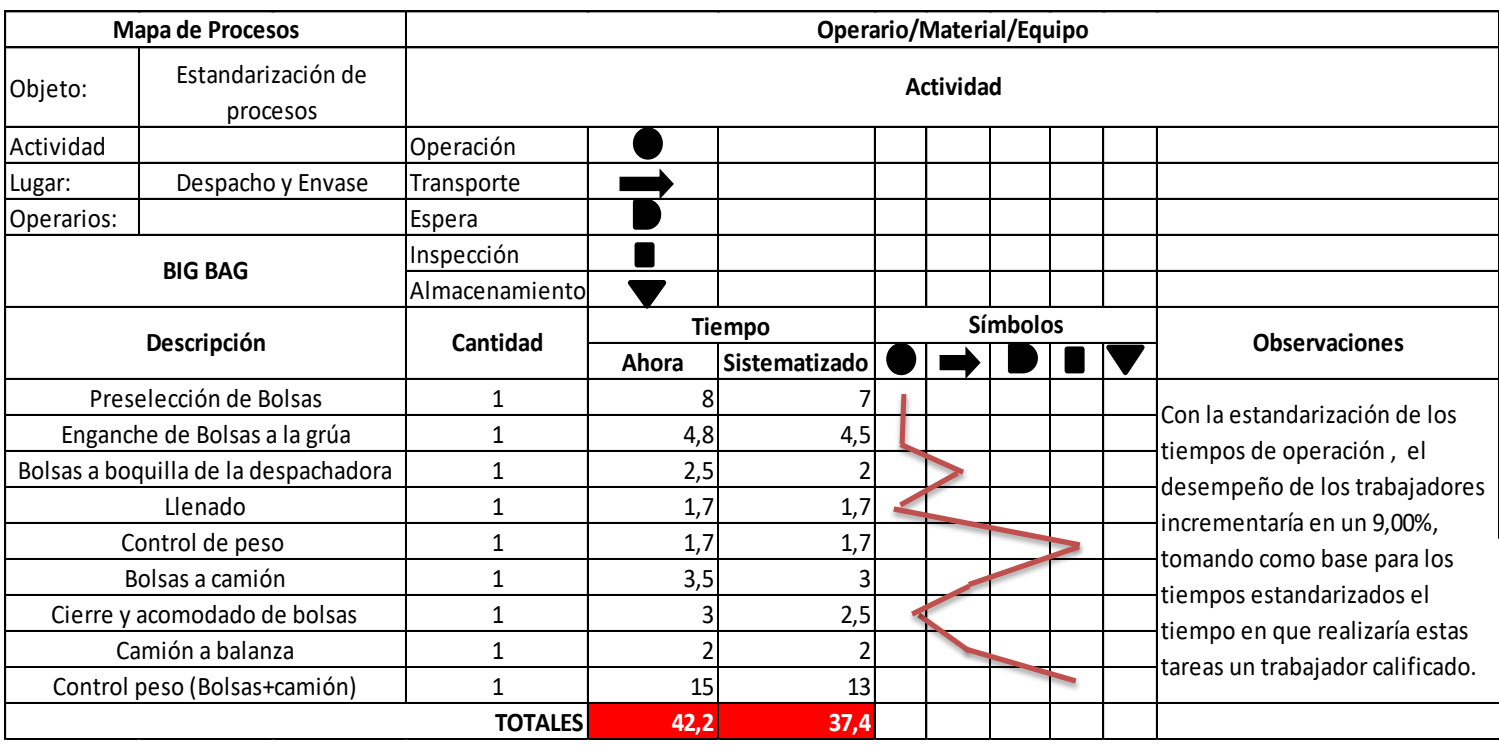

Gráfico 4. Mapa de procesos para la modalidad big bag.

Como se muestra en el gráfico 4, el desempeño de los trabajadores mejorará en un $9 \%$ con la estandarización de los tiempos de operación. Es importante mencionar que para la medición de tiempos (en las dos modalidades), se consideró que las actividades serán realizadas por un operario calificado, por lo que es necesario considerar una capacitación de todo el personal del área antes de poner en marcha esta estandarización, de tal forma que se alcancen los tiempos propuestos.

\section{CONCLUSIONES}

Tomando como objeto de estudio al área de despacho de FANCESA, se contrastó la teoría con la práctica y se comprobó que existe correlación inversa entre productividad y tiempos de operación, y correlación directa entre productividad y mantenimiento preventivo. Sin embargo, en este contexto particular, se descartó la interrelación entre productividad y condiciones de trabajo. Se concluye entonces, que no necesariamente en todos los contextos las condiciones de trabajo afectarán la productividad, sino más bien, el estudio realizado indica que mejorar las condiciones de trabajo en el área de despacho y envasado de FANCESA, no logra elevar la productividad del área.

Gracias a este nuevo saber científico, se propuso dos acciones de optimización de tiempos de producción para incrementar la 
productividad del sector de despacho en la modalidad de ensacado y big bag: la implementación de un programa de mantenimiento mecánico preventivo y la estandarización de procesos del área. Se concluye entonces que, al aplicar estas acciones es posible reducir los tiempos de operación, causando un efecto positivo en la productividad del área. Demostrando que un mantenimiento deficiente es una de las principales causantes de tiempos improductivos, ya que provoca paradas imprevistas por fallas en la maquinaria y equipo.

\section{REFERENCIAS}

Andrade, A; Del Río, C; Daissy A. (2018). Estudio de Tiempos y Movimientos para Incrementar la Eficiencia en una Empresa de Producción de Calzado. Ecuador

FANCESA. (2015) Memorias 2015. Bolivia

FANCESA. (2019) Memorias 2019. Bolivia

García, M; Quispe, C; Ráez, L. (2007). Mejora continua de la calidad en los procesos.
Gonzales, A. (2014) Mejora del Proceso de Despacho de Bolsas de Cemento en Unacem. Lima

Heizer, J; Render B. (2009). Principio de la Administración de Operaciones. Edición 7. México

Kanawaty, G. (2012). Introducción al Estudio del Trabajo. 4ta Edición.

Sira, S. (2011). Aplicación tecnológica del Diagrama - Máquinas. Ingeniería UC. Vol. 18; Núm. 3; pp 17-28

Ruiz, J. (2013). Productividad e Incentivos: Cómo hacer que los tiempos de fabricación se cumplan

Marcombo, E. (2015). Estudio de tiempos y movimientos para la mejora de la productividad en la empresa Productos del día dedicada a la fabricación de balanceado avícola

Morillo Santa Cruz, T; Lozano, M. (2007). Estudio de la productividad en una obra de edificación 